\title{
PARÂMETROS FITOTÉCNICOS DE UMA CULTURA DO TRIGO (Triticum aestivum L.) COM ADUBAÇÃO NITROGENADA EM COBERTURA, EM COLOMBO - PR
}

\author{
Phytotechnical Parameters of Wheat (Triticum aestivum L.) Crop \\ with Cover Nitrogen Fertilization, in Municipality of Colombo - PR
}

Fabrício M. Schmidt

Aluno de Agronomia da PUCPR, Centro de Ciências Agrárias e Ambientais, São José dos Pinhais, PR. E-mail: bito_ms@hotmail.com.

Flora Osaki

Engenheira Agrônoma, Professora Adjunta da PUCPR, Centro de Ciências Agrárias e Ambientais, São José dos Pinhais, PR. E-mail: rk_osaki@netpar.com.br.

\begin{abstract}
Resumo
O trigo (Triticum aestivum L.) é uma das principais culturas no Brasil e a quantidade de nitrogênio a ela aplicada é um dos fatores que mais estimula sua produção, o que resulta num maior número de perfilhos, portanto maior número de plantas $/ \mathrm{m}^{2} \mathrm{e}$, conseqüentemente, maior produtividade em $\mathrm{kg} / \mathrm{ha}$. O presente trabalho teve como principal objetivo avaliar os efeitos de adubação de nitrogênio em cobertura em relação ao número de espigas $/ \mathrm{m}^{2}$, número de espiguetas por espigas, peso hectolitro, peso de 1000 grãos e produtividade da cultivar IPR118. O experimento foi conduzido na fazenda WS, localizada no município de Colombo - PR, no período de 10/04/2006 a 20/10/2006. O delineamento utilizado foi o de blocos ao acaso com 8 tratamentos e 4 repetições, sendo utilizado uréia como adubo de cobertura nas seguintes dosagens $0,20,40,80,160,250,350$ e $450 \mathrm{~kg} / \mathrm{ha}, \mathrm{T} 1, \mathrm{~T} 2, \mathrm{~T} 3, \mathrm{~T} 4, \mathrm{~T} 5, \mathrm{~T} 6, \mathrm{~T} 7 \mathrm{e}$ T8, respectivamente. O maior rendimento foi obtido com o tratamento T6 (1445 kg/ha), entretanto esse não diferiu estatisticamente apenas do tratamento T8 (1436 kg/ha). O maior número de espigas $/ \mathrm{m}^{2}$ foi obtido no tratamento T6 (96), não diferindo estatisticamente apenas do tratamento T7 $(94,5)$. A análise de regressão mostrou um decréscimo no rendimento e número de espigas $/ \mathrm{m}^{2}$ a partir da dose de $250 \mathrm{~kg}$ de N/ha. Não houve diferenças significativas quanto ao peso de 1000 grãos e peso hectolitro, porém no período do experimento ocorreu uma grande estiagem na época de germinação e geadas durante o florescimento.
\end{abstract}

Palavras-chave: Triticum aestivum L.; Nitrogênio; Adubação em cobertura; Produtividade. 


\begin{abstract}
The wheat (Triticum aestivum L.) is one of the main crops of Brazil and the quantity of nitrogen used in its crop is one of the main factors that stimulate its production, what results in higher number of stems, consequently a higher number of plants $/ \mathrm{m}^{2}$ and, therefore, a higher productivity in $\mathrm{kg} / \mathrm{ha}$. The present research had as its main objective to evaluate the cover nitrogen fertilization effect regarding the number of spikes $/ \mathrm{m}^{2}$, number of ears in each spike, hectoliter weight, 1000 grains weight and the yield of the cultivar IPR118. The experiment was conducted in WS farm, located in the municipality of Colombo, PR, between 10/04/2006 and 20/10/2006. A randomized block design was used, with 8 treatments and 4 replications, with urea as cover fertilization in doses: 0, 20,40,80,160,250, 350 and $450 \mathrm{~kg} / \mathrm{ha}, \mathrm{T1}, \mathrm{T2}, \mathrm{T3}, \mathrm{T4}, \mathrm{T5}, \mathrm{T6}, \mathrm{T} 7$ and T8, respectively. The highest yield was obtained with the T6 treatment (1445 $\mathrm{kg} / \mathrm{ha}$ ), however this did not differ statistically only from the T8 treatment (1436 $\mathrm{kg} / \mathrm{ha}$ ). The highest number of spikes $/ \mathrm{m}^{2}$ was obtained in the T6 treatment (96), not differing statistically only from the $T 7$ treatment (94.5). The regression analysis showed a decrease of the yield and number of spikes $/ \mathrm{m}^{2}$ above the $250 \mathrm{~kg}$ of $\mathrm{N} / \mathrm{h}$ a dose. It did not show significant differences for the 1000 grains weight and hectoliter weight, however in the period of the experiment a long drought occurred during the germination period and frosts during the bloom.
\end{abstract}

Keywords: Triticum aestivum L.; Nitrogen; Cover fertilization; Yield.

\title{
INTRODUÇÃO
}

A produção estimada para o trigo no ano de 2005 foi de 4,896 milhões de toneladas, tendo o Paraná como seu maior produtor, com uma representação de $56 \%$ e uma produção de 2,7 milhões de toneladas de grãos (RCCBPT, 2004; SEAGRI, 2006).

O trigo é a segunda cultura de grãos em nível mundial de produção, por isso a pesquisa tem destinado tempo, recursos humanos e financeiros no desenvolvimento de tecnologia para atingir produções elevadas.

Nas recomendações técnicas para a cultura, a adubação está diretamente relacionada à quantidade de adubo aplicada. O nitrogênio é um dos elementos mais absorvidos pela cultura e pode também ser o mais limitante. Estudos mostram que para a produção de três toneladas de grãos, são necessários $56 \mathrm{~kg}$ de nitrogênio, diferentes doses de nitrogênio e elevadas populações de plantas são utilizadas, visando obter altas produtividades em trigo. O rendimento do trigo resulta da escolha da cultivar, disponibilidade de água, da quantidade de insumos e das técnicas de manejo empregadas. A crescente utilização de cultivares de alto potencial produtivo tem implicado no uso mais freqüente de insumos, entre os quais a adubação nitrogenada (ZAGONEL, 2002).

$\mathrm{Na}$ determinação da resposta do trigo à adubação nitrogenada é importante considerar o comportamento da cultura para diferentes doses do fertilizante aplicado. Sari et al. (1997) estudaram quatro doses de adubo nitrogenado para o trigo cultivado em sistema de plantio direto $(0,30 \mathrm{~kg} / \mathrm{ha}, 60 \mathrm{~kg} /$ ha e $90 \mathrm{~kg} / \mathrm{ha}$ ) e verificaram a maior produção para a dose de $60 \mathrm{~kg} / \mathrm{ha}$.

Em um experimento com diferentes níveis de nitrogênio para o trigo (zero, 30, $60 \mathrm{~kg} / \mathrm{ha}$ ) em três épocas (pré-semeadura, semeadura e em cobertura) foram verificadas maiores produtividades de grãos com a utilização de doses mais elevadas de N, $795 \mathrm{~kg} / \mathrm{ha}$ e $1240 \mathrm{~kg} / \mathrm{ha}$ para 30 e $60 \mathrm{~kg}$ de N/ha, respectivamente (POTAFOS, 1996).

\section{MATERIAL E MÉTODOS}

O experimento foi conduzido na fazenda WS, localizada no município de Colombo, região metropolitana de Curitiba. A cultivar escolhida foi a IPR 118, de ciclo precoce, altura da planta de $82 \mathrm{~cm}$ e moderadamente tolerante ao alumínio. 
A análise de solo constou de uma amostra composta, não havendo necessidade de calagem ( $\mathrm{pH}$ de 6,25). A semeadura foi feita em meados de maio, espaçamento entrelinhas de $20 \mathrm{~cm}$, profundidade de $5 \mathrm{~cm}$ e 60 sementes $/ \mathrm{m}$ correspondendo a 300 plantas por metro quadrado.

A adubação para fósforo, potássio e nitrogênio de base foi feita na semeadura, utilizando-se o super triplo, cloreto de potássio e uréia, na ordem de $60,80,50 \mathrm{~kg} \mathrm{ha}^{-1}$ de acordo com os resultados da análise de solo, seguindo as recomendações para o Estado do Paraná.

O delineamento foi o de blocos ao acaso, com oito tratamentos (oito níveis de nitrogênio em cobertura: 0, $20 \mathrm{~kg} / \mathrm{ha}, 40 \mathrm{~kg} / \mathrm{ha}, 80 \mathrm{~kg} / \mathrm{ha}, 160 \mathrm{~kg} / \mathrm{ha}, 250 \mathrm{~kg} / \mathrm{ha}, 350 \mathrm{~kg} / \mathrm{ha}$ e $450 \mathrm{~kg} / \mathrm{ha})$ e quatro repetições.

A adubação em cobertura foi feita no início do perfilhamento. O adubo nitrogenado de cobertura utilizado foi a uréia.

A parcela teve $6,0 \mathrm{~m} \times 2,5 \mathrm{~m}$ e uma área de parcela total de $15,00 \mathrm{~m}^{2}$. A área útil foi de 1,8 m x 5,0 m perfazendo a área útil para a amostragem de $9 \mathrm{~m}^{2}$. Entre as parcelas foi mantida uma distância de $0,5 \mathrm{~m}$.

Os parâmetros avaliados foram:

a) Número de espigas por metro quadrado, coletando-se todas as plantas em uma área de 1,0 $\mathrm{m}^{2}$, colocadas em sacos de papel, identificadas de acordo com o tratamento e encaminhadas ao Laboratório de Solos da Pontifícia Universidade Católica do Paraná, São José dos Pinhais, onde foi feita a contagem das espigas e a tabulação dos dados.

b) Peso do hectolitro ( $\mathrm{kg} / \mathrm{hl}$ ): foi medido em um recipiente de 11 (litro) e transformado em $\mathrm{kg} /$ hl pela tabela oficial de peso hectolitro para cereais.

c) Peso de 1000 grãos (g): para a determinação foi coletado o material a campo e colocado em sacos de papel, identificados e levados para o laboratório. As amostras de grãos de trigo foram pesadas em balança de precisão com aproximação de 0,0001 g e capacidade total para $5 \mathrm{~kg}$.

A fórmula utilizada para o cálculo de 1000 grãos foi a seguinte:

Peso de 1000 grãos $(\mathrm{g})=\frac{\text { peso da amostra }(\mathrm{g})-1000}{\text { número de sementes da amostra }}$

d) Rendimento ( $\mathrm{kg} / \mathrm{ha})$

Foi realizada a colheita na área útil $\left(4 \mathrm{~m}^{2}\right)$ de cada parcela para os diferentes tratamentos. O material coletado foi debulhado e em seguida foi determinada a umidade do grão. Foi utilizada a fórmula do desconto de umidade para se obter 13\% de umidade.

Os resultados foram submetidos à análise de variância e as variáveis que se apresentaram homogêneas foram avaliadas pelo teste de Tukey a 5\% de significância. Além da comparação de médias pelo teste de Tukey, os resultados foram submetidos à análise de regressão.

\section{RESULTADOS E DISCUSSÃO}

Os resultados obtidos são apresentados na Tabela 1. 
TABELA 1 - Número de espigas, peso hectolitro, peso de 1000 grãos, rendimento da cultura do trigo (Triticum aestivum L.)

Table 1 - Number of spikes, hectoliter weight, 1000 grains weight, wheat

(Triticum aestivum L.) crop yield

\begin{tabular}{|c|c|c|c|c|c|c|c|c|c|c|}
\hline \multirow[b]{2}{*}{ Variável } & \multicolumn{10}{|c|}{ DOSES DE N kg/ha } \\
\hline & $\begin{array}{c}\mathrm{T} 1 \\
\mathbf{0}\end{array}$ & $\begin{array}{l}\mathrm{T} 2 \\
20\end{array}$ & $\begin{array}{l}\text { T3 } \\
40\end{array}$ & $\begin{array}{l}\text { T4 } \\
80\end{array}$ & $\begin{array}{l}\text { T5 } \\
160\end{array}$ & $\begin{array}{l}\mathrm{T} 6 \\
250\end{array}$ & $\begin{array}{l}\mathrm{T} 7 \\
350\end{array}$ & $\begin{array}{l}\mathrm{T} 8 \\
450\end{array}$ & $\begin{array}{l}\mathrm{dms} \\
5 \%\end{array}$ & $\begin{array}{c}\mathrm{CV} \\
\%\end{array}$ \\
\hline $\begin{array}{l}\mathrm{N} .^{\circ} \text { de } \\
\text { espigas } / \mathrm{m}^{2}\end{array}$ & $\begin{array}{c}60,8 \\
\mathrm{e}\end{array}$ & $\begin{array}{c}63,0 \\
\text { de }\end{array}$ & $\begin{array}{c}65,5 \\
d\end{array}$ & $\begin{array}{c}70,5 \\
c\end{array}$ & $\begin{array}{c}90,0 \\
\mathrm{~b}\end{array}$ & $\begin{array}{c}96,0 \\
\mathrm{a}\end{array}$ & $\begin{array}{c}94,5 \\
\mathrm{a}\end{array}$ & $\begin{array}{c}90,5 \\
\mathrm{~b}\end{array}$ & 4,86 & 1,50 \\
\hline $\begin{array}{l}\text { Peso } \\
\text { hectolitro } \\
\mathrm{kg} / \mathrm{hl}\end{array}$ & $\begin{array}{c}73,64 \\
a\end{array}$ & $\begin{array}{c}74,09 \\
\mathrm{a}\end{array}$ & $\begin{array}{c}73,36 \\
a\end{array}$ & $\begin{array}{c}74,70 \\
\mathrm{a}\end{array}$ & $\begin{array}{c}73,53 \\
a\end{array}$ & $\begin{array}{c}74,54 \\
\mathrm{a}\end{array}$ & $\begin{array}{c}73,08 \\
\mathrm{a}\end{array}$ & $\begin{array}{c}74,14 \\
\mathrm{a}\end{array}$ & 2,17 & 1,23 \\
\hline $\begin{array}{l}\text { Peso de } \\
1000 \text { grãos g }\end{array}$ & $\begin{array}{c}36,25 \\
a\end{array}$ & $\begin{array}{c}35,50 \\
\mathrm{a}\end{array}$ & $\begin{array}{c}37,75 \\
a\end{array}$ & $\begin{array}{c}37,75 \\
\mathrm{a}\end{array}$ & $\begin{array}{c}36,50 \\
\mathrm{a}\end{array}$ & $\begin{array}{c}37,75 \\
a\end{array}$ & $\begin{array}{c}38,00 \\
\mathrm{a}\end{array}$ & $\begin{array}{c}36,00 \\
\mathrm{a}\end{array}$ & 2,82 & 5,52 \\
\hline $\begin{array}{l}\text { Rendim } \\
\mathrm{kg} / \mathrm{ha}\end{array}$ & $\begin{array}{c}894,0 \\
\mathrm{f}\end{array}$ & $\begin{array}{c}909,0 \\
\mathrm{f}\end{array}$ & $\begin{array}{c}1014,0 \\
\mathrm{e}\end{array}$ & $\begin{array}{c}1219,0 \\
\mathrm{~d}\end{array}$ & $\begin{array}{c}1307,0 \\
\text { c }\end{array}$ & $\begin{array}{c}1445,0 \\
\mathrm{a}\end{array}$ & $\begin{array}{c}1416,0 \\
b\end{array}$ & $\begin{array}{c}1436,0 \\
\mathrm{ab}\end{array}$ & 25,32 & 0,88 \\
\hline
\end{tabular}

Médias seguidas pela mesma letra não diferem entre si estatisticamente.

Os resultados na Tabela 1 mostraram que para o peso hectolitro e a massa de 1000 grãos não apresentaram diferença significativa pelo teste F $(p>0,05)$, o que significa que a dose de nitrogênio não interferiu nesses aspectos. Didonet (2000) também constatou que não houve diferença no peso de 1000 grãos entre diferentes doses de nitrogênio para a cultura do trigo. Cánovas e Trindade (2003) verificaram uma tendência decrescente de peso hectolitro para duas variedades de trigo (EMBRAPA 22; EMBRAPA 42) em cinco níveis de adubação em cobertura com nitrogênio (0 kg/ha, $50 \mathrm{~kg} / \mathrm{ha}, 100 \mathrm{~kg} / \mathrm{ha}, 150 \mathrm{~kg} / \mathrm{ha}$ e $200 \mathrm{~kg} / \mathrm{ha}$ ). Os mesmos autores não encontraram diferenças estatísticas significativas para o peso de 1000 grãos nas doses estudadas, mas relataram tendência de redução no peso com o aumento das doses de nitrogênio.

Analisando a quantidade de espigas por $\mathrm{m}^{2}$ Figura 1, a adubação proporcionou diferentes respostas à cultura do trigo. Observa-se, na Figura 1, a tendência de incremento no número de espigas/ $\mathrm{m}^{\prime \prime}$ até $250 \mathrm{~kg} / \mathrm{ha}$ de $\mathrm{N}$, ocorrendo um decréscimo a partir dessa dose.

Os tratamentos $6(250 \mathrm{~kg} / \mathrm{ha})$ e $7(350 \mathrm{~kg} / \mathrm{ha})$, nessa ordem, foram os que apresentaram maior número de espigas $/ \mathrm{m}^{2}$, não diferindo estatisticamente entre si pelo teste $\mathrm{F}(p>0,05)$. O tratamento 1 , ausência de nitrogênio, apresentou a menor quantidade de espigas $/ \mathrm{m}^{2}$, o qual não diferiu estatisticamente do tratamento 2 (20 kg de N/ha). Já os tratamentos $5(160 \mathrm{~kg} / \mathrm{ha})$ e $8(450 \mathrm{~kg} / \mathrm{ha})$ não apresentaram diferença estatisticamente significativa entre si e apresentaram número de espigas $/ \mathrm{m}^{2}$ inferior aos tratamentos 6 e 7. Em trabalho realizado por Marchetti, Caramori e Campos (2001), com o objetivo de estudar o efeito de diferentes níveis de $\mathrm{N}$ para a cultura de trigo, verificou-se um acréscimo no número de espigas com a adição de níveis crescentes de nitrogênio. Já no presente trabalho, os resultados obtidos mostraram um aumento no número de espigas até $250 \mathrm{~kg}$ de N/ha e a tendência de decréscimo após esta dose conforme Figura 1. 


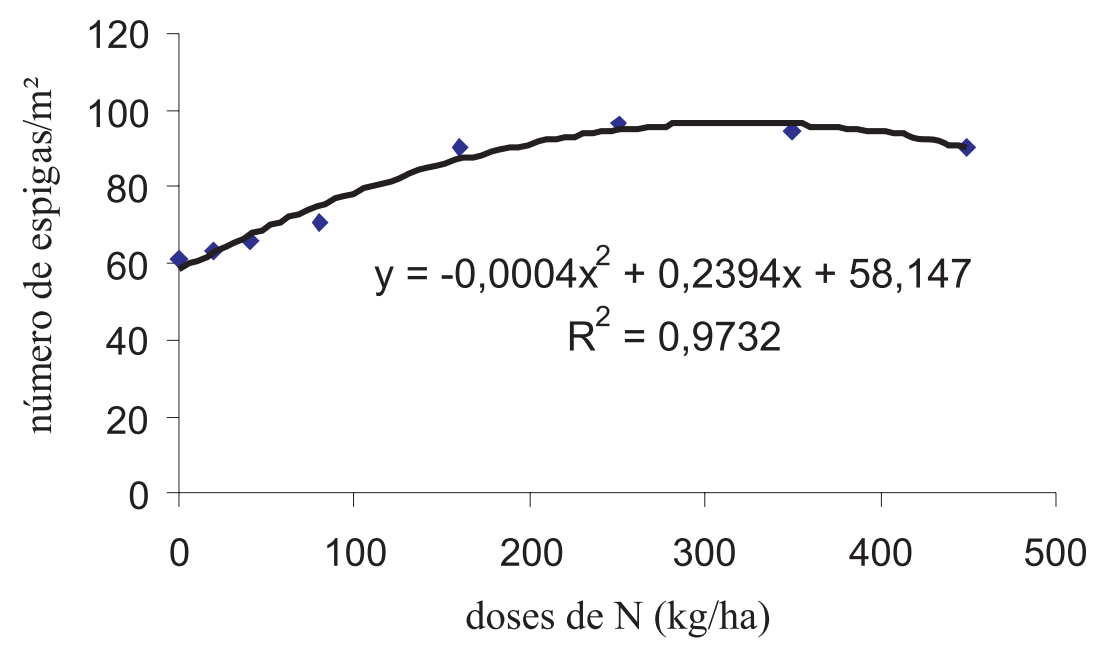

FIGURA 1 - Número de espigas/m² em diferentes níveis de nitrogênio em cobertura para a cultura de trigo

Figure 1 - Number of spikes $/ m^{2}$ in wheat crop with different levels of nitrogen in cover fertilization

A exigência de nitrogênio está relacionada com a velocidade de crescimento, com a densidade de plantas, das condições climáticas e dos teores dos demais nutrientes presentes no solo (MALAVOLTA, 1981).

Atualmente, alguns autores discutem modelos para sustentar o conceito de percentagem crítica de nitrogênio nas culturas. Dessa forma, as práticas de adubação que visam fornecer $\mathrm{N}$ de modo a promover a máxima produção de matéria seca podem resultar em situações de deficiência do nutriente ou de consumo de luxo. O primeiro resulta em perdas de produtividade e, o segundo, além da redução na produção, em aumento de custo para o produtor e contaminação do meio (OSAKI, 2000; WHITEHEAD, 1995).

Cánovas e Trindade (2003) verificaram incremento no rendimento da cultura do trigo quando aplicados 0, 50, 100, 150 e $200 \mathrm{~kg}$ de N/ha. Relataram ainda que a máxima eficiência técnica correspondeu à aplicação de $149 \mathrm{~kg}$ de N/ha para a cultivar EMBRAPA 22 e $205 \mathrm{~kg}$ de N/ha para a cultivar EMBRAPA 42.

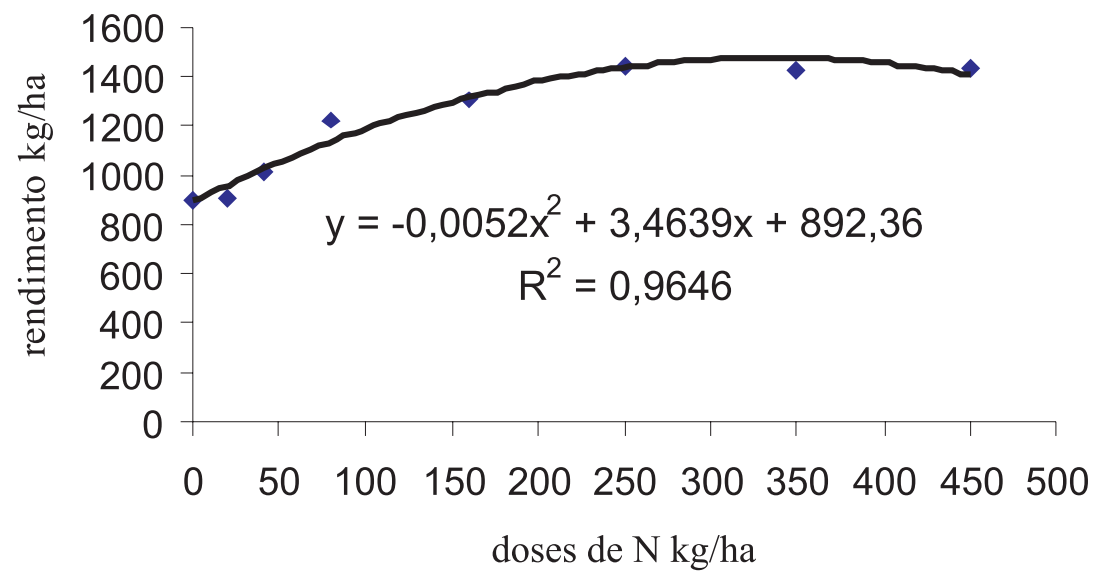

FIGURA 2 - Rendimento da cultura de trigo (Triticum aestivum L.) com diferentes doses de nitrogênio em cobertura

Figure 2 - Wheat (Triticum aestivum L.) crop yield with different nitrogen doses in cover fertilization 
Analisando a Tabela 1, pode-se observar que os tratamentos $1(0 \mathrm{~kg} / \mathrm{ha})$ e $2(20 \mathrm{~kg} / \mathrm{ha})$ tiveram os menores rendimentos: 894 e $909 \mathrm{~kg} / \mathrm{ha}$, respectivamente. O maior rendimento foi observado para $\mathrm{O}$ tratamento 6 (250 kg/ha de N), que não diferiu estatisticamente do tratamento 8 (450 kg/ha), sendo suas médias de produtividade de 1445 e $1436 \mathrm{~kg} / \mathrm{ha}$, respectivamente.

O IPR 118 apresentou bom rendimento, com potencial produtivo que varia de 3 mil a 5 mil kg/ ha, sendo que a melhor produtividade obtida neste trabalho foi de $1.445 \mathrm{~kg} / \mathrm{ha}$, bem abaixo do rendimento relatado para a cultura. Provavelmente este baixo rendimento deve-se ao longo período de estiagem na região (IAPAR- 2004).

Na Figura 2 observa-se que houve tendência de aumento no rendimento até a dose de $250 \mathrm{~kg} /$ ha, com um decréscimo a partir desse ponto. Resultados semelhantes foram relatados em experimento realizado em vasos por Marchetti, Caramori e Campos (2001). Os autores relataram para doses maiores de $\mathrm{N}$ uma queda de produção, indicando que níveis mais altos de $\mathrm{N}$ podem ter provocado um desequilíbrio entre os nutrientes na solução nutritiva.

No caso de experimentos de adubação nitrogenada para o trigo, observou-se que podem ser obtidas respostas diferenciadas, pois o rendimento pode ser influenciado pela cultivar utilizada (OLIVEIRA et al., 1981).

\section{CONCLUSÕES}

Nas doses testadas de adubo nitrogenado não houve diferenças estatísticas para o peso hectolitro.

O maior número de espigas $/ \mathrm{m}^{2}$ foi obtido com a dose de $250 \mathrm{~kg} / \mathrm{ha}(96,0)$, porém não diferiu estatisticamente apenas do tratamento com aplicação de $350 \mathrm{~kg}$ de N/ha.

A análise de regressão mostrou um decréscimo no número de espigas $/ \mathrm{m}^{2}$ a partir de $250 \mathrm{~kg}$ de $\mathrm{N} / \mathrm{ha}$, com $\mathrm{R}^{2}$ de 0,97 .

O maior rendimento (1445 kg/ha) foi obtido no tratamento com dose $250 \mathrm{~kg}$ de N/ha, que não diferiu estatisticamente apenas do tratamento com aplicação de $450 \mathrm{~kg}$ de N/ha $(1436 \mathrm{~kg} / \mathrm{ha})$.

A análise de regressão mostrou tendência de decréscimo da produtividade a partir de $250 \mathrm{~kg}$ de $\mathrm{N} / \mathrm{ha}$, com $\mathrm{R}^{2}$ de 0,96 , porque aplicações nitrogenadas acima dessa dosagem não contribuem para incrementos de produtividade.

\section{REFERÊNCIAS}

CÁNOVAS, A. D; TRINDADE, M. G. Efeito de níveis de nitrogênio e freqüência de aplicação de água na produtividade e na aptidão industrial do trigo. Comunicado Técnico 70. Santo Antônio de Goiás, dez. 2003.

DIDONET, A. D. et al. Realocação de nitrogênio e de biomassa para os grãos, em trigo submetido a inoculação de Azospirillum. Pesq. Agropec. Bras., v. 35, n. 2, p. 401-411, fev. 2000.

IAPAR - PR. Coopavel. 2004. Disponível em: <http://www.iapar.br/noticias/coopavel.html>. Acesso em: 24 fev. 2006.

MALAVOLTA, E. Manual de química agrícola adubos e adubação. 3. ed. São Paulo: Ceres, 1981. p. 3196.

MARCHETTI, M. E.; CARAMORI, T. B. A.; CAMPOS, A. M. B. Resposta de duas espécies de trigo ao nitrogênio e ao fósforo em solução nutritiva. Ciência Agrot., Lavras, v. 25, n. 4, p. 925-933, jul./ago., 2001.

OLIVEIRA, E. F.; FRANCO, F. A.; BEGO, A. Efeito de doses e épocas de aplicação de nitrogênio para duas cultivares de trigo (Triticum aestivum L.) IAC 5 e Nambu. In: Organização das cooperativas do Estado do Paraná. Resultados de pesquisa com trigo e triticale nos anos de 1979 e 1980. Cascavel, 1981. p. 180186. 
OSAKI, F. Índice de nutrição nitrogenada na cultura de azevém (Lolium multiflorum Lam.) para a região metropolitana de Curitiba). Boletim Técnico n. 62. IAPAR, maio, 2000. p. 18.

POTAFOS. Nutri-fatos: informação agronômica sobre nutrientes para as culturas. Arquivo do Agrônomo, n. 10, mar. 1996.

REUNIÃO DA COMISSÃO CENTRO-SUL BRASILEIRA DE PESQUISA DE TRIGO, 19. 2004. Londrina, PR. Reunião da Comissão Brasileira de Pesquisa de Triticale, 10., 2004. Londrina, PR. Informações técnicas das comissões centro-sul brasileira de pesquisa de trigo e de triticale para a safra de 2004. Londrina, 2004. p. 214.

SARI, Y. et al. Ligh691 t 692 and electron microscopic immunocytochemical visualization of 5-HT1B receptors in the rat 693 brain. Brain Res., v. 760, n. 281, p. 281-286, 1997.

SEAGRI. Secretaria de Agricultura, Irrigação e Reforma Agrária. Cultura do Trigo. Disponível em: <http:/ /www.bahia.ba.gov.br/seagri/Trigo.htm>. Acesso em: 30 mar. 2006.

WHITEHEAD, D. C. Grassland nitrogen. CAB INTERNATIONAL Wallingford, OxOn OX10 8DE, UK. 1995.

ZAGONEL, J. et al. Doses de nitrogênio e densidades de plantas com e sem um regulador de crescimento afetando o trigo, cultivar OR-1. Cienc. Rural, v. 32, n. 1, p. 25-29, fev. 2002. 